\title{
The risk of perchlorate and iodine on the incidence of thyroid tumors and nodular goiter: a case-control study in southeastern China
}

Huirong Wang ${ }^{1,2}$, Yousheng Jiang ${ }^{1}$, Jiayi Song ${ }^{1}$, Huiwen Liang ${ }^{1}$, Yuan Liu', Jiewu Huang ${ }^{1}$, Pengliang Yin ${ }^{1}$, Dongting Wu ${ }^{1,3}$, Hang Zhang ${ }^{1}$, Xinjie Liư ${ }^{4}$, Dongxian Zhou ${ }^{4}$, Wei Wei ${ }^{5}$, Lin Lei ${ }^{6}$, Ji Peng ${ }^{6}$ and Jianqing Zhang ${ }^{1 *}$ (D)

\begin{abstract}
Background: The incidence rates of thyroid tumors and nodular goiter show an upward trend worldwide. There are limited reports on the risk of perchlorate and iodine on thyroid tumors, but evidence from population studies is scarce, and their impact on thyroid function is still uncertain. Therefore, the objective of this study was to investigate the association of perchlorate and iodine with the risk of nodular goiter (NG), papillary thyroid microcarcinoma (PTMC), and papillary thyroid carcinoma (PTC) and to assess the correlation between perchlorate and iodine with thyroid function indicators.
\end{abstract}

Methods: A case-control population consisting of 184 pairs of thyroid tumors and nodular goiter matched by gender and age ( \pm 2 years) was recruited in this study. Serum and urine samples were collected from each participant. Thyroid function indicators in serum were tested by automatic chemical immunofluorescence, and perchlorate and iodine levels in urine were determined by ultra-high performance liquid chromatography tandem-mass spectrometry and inductively coupled plasma-mass spectrometry, respectively. Conditional logistic regressions and multiple linear regressions were used to analyze the associations.

Results: Urinary perchlorate concentration was significantly higher in total cases, NG and PTC than in the corresponding controls $(P<0.05)$. Perchlorate was positively associated with PTC $(\mathrm{OR}=1.058,95 \% \mathrm{Cl}: 1.009,1.110)$ in a non-linear dose-response relationship, but there was no association between perchlorate and NG or PTMC. lodine was not associated with the risk of thyroid tumors and NG and did not correlate with the thyroid function indicators. Furthermore, perchlorate showed a positive correlation with thyroid stimulating hormone (TSH) at iodine adequate levels $(P<0.05)$, and a negative correlation with free triiodothyronine (FT3) and a positive correlation with thyroglobulin antibody $(\mathrm{TgAb})$ at iodine more than adequate or excess levels $(P<0.05)$.

Conclusions: Perchlorate can increase the risk of PTC in a non-linear dose-response relationship and disturb the thyroid hormone homeostasis and thyroid autoantibody levels.

*Correspondence: 969676617@qq.com

${ }^{1}$ Shenzhen Center for Disease Control and Prevention, No.8 Longyuan

Road, Nanshan District, Shenzhen 518055, China

Full list of author information is available at the end of the article original author(s) and the source, provide a link to the Creative Commons licence, and indicate if changes were made. The images or other third party material in this article are included in the article's Creative Commons licence, unless indicated otherwise in a credit line to the material. If material is not included in the article's Creative Commons licence and your intended use is not permitted by statutory regulation or exceeds the permitted use, you will need to obtain permission directly from the copyright holder. To view a copy of this licence, visit http://creativecommons.org/licenses/by/4.0/. The Creative Commons Public Domain Dedication waiver (http://creativeco mmons.org/publicdomain/zero/1.0/) applies to the data made available in this article, unless otherwise stated in a credit line to the data. 


\section{Highlights}

- Patients with thyroid tumor have higher perchlorate exposure than control.

- Perchlorate can increase the risk of papillary thyroid carcinoma.

- Perchlorate can disturb the homeostasis of thyroid function.

- Perchlorate was positively correlated with thyroglobulin antibody.

Keywords: Perchlorate, lodine, Thyroid cancer, Nodular goiter, Thyroid function

\section{Background}

The incidence of thyroid tumor has been sharply growing around the world, identification and characterization the risk factors for thyroid cancer and nodules have become a scientific hot topic in recent years. Thyroid tumors are divided into benign thyroid adenoma and malignant thyroid cancer (TC), and thyroid nodules (TNs) are a common benign proliferative disease of the thyroid gland with the detection rate in the general population as high as $65 \%$ [1]. Studies have shown that about $10 \%$ of TNs have the potential to progress to TC [1]. TC is the most common malignant tumor of the endocrine system and includes papillary carcinoma, follicular carcinoma, undifferentiated carcinoma, and medullary carcinoma. According to the 'GLOBOCAN 2020' published by the International Association of Cancer Registries (IARC) and World Health Organization (WHO), there were 586,202 new cases of global TC in 2020, of which 59.7\% were in Asia [2]. Moreover, the total incidence of TC in the United States increased at a rate of 3\% per year from 1974 to 2013 [3], and the number of TC cases in Denmark tripled between 1980 and 2014 [4]. Similarly, the age-standardized incidence of TC in China increased from $3.21 / 100,000$ to $9.61 / 100,000$ at an average rate of $3 \%$ per year from 2005 to 2015 [5]. Furthermore, the incidence rate of TC in Shenzhen, a typical fast modern and urbanized city of China, increased at an average rate of $11.33 \%$ per year from 2001 to 2015 ; the world population standard incidence rate increased from $3.55 / 100,000$ to $17.97 / 100,000$, ranking TC from the tenth to the second most common malignant tumor [6]. Although the incidence of TC has increased worldwide, the mortality rate is on a stable or even decreasing trend with the improvement of diagnostic and treatment techniques and early intervention of the disease [7]. Studies have shown that thyroid disease management costs in the United States are expected to triple by up to $\$ 3.5$ billion/year by 2030 , thus TC brings a great health and economic burden to human society [7]. There is an urgent need to explore the risk factors and potential environmental chemicals related to thyroid tumors so that thyroid disease can be controlled and prevented around the world.
It has been suggested that multiple factors, including genetics, radiation, iodine, autoimmune diseases, and complex and diverse environmental factors, such as environmental chemicals, may be the risk factors for thyroid tumors [8]. It has been considered that the rapid increase in the incidence of $\mathrm{TC}$ is the result of high-resolution ultrasound diagnostic methods and overdiagnosis. However, the effects of iodine intake and environmental pollutants such as perchlorate on thyroid tumors and their interactions cannot be ignored, but the evidence for such effects remains scarce. Perchlorate is mainly used for the production of rocket fuel and fireworks as oxidants in explosives [9]; with its high water solubility, stability, and difficulty to degrade, it is widely present in water, soil, air, and crops [10]. Most people are exposed to perchlorate through the dietary route $[11,12]$. Industry wastewater and domestic sewage are the main sources of widespread perchlorate pollution in China, and most of the water and food samples (such as rice, milk, purified water) collected in China contain perchlorate in varying degrees $[13,14]$. Previous studies have found that people living in the fireworks production area and electronic waste recycling area have higher concentrations of perchlorate in body fluids $[9,15]$. While iodine plays an important role in the functioning of the thyroid gland and the thyroid hormone secretion system, perchlorate is structurally similar to iodide and is a competitive iodide inhibitor that can reduce iodine utilization by the thyroid gland $[16,17]$. It has been demonstrated that perchlorate may lead to thyroid dysfunction or thyroid diseases by mediating the active transport of $\mathrm{I}^{-}$on plasma membrane glycoprotein, thereby preventing $\mathrm{I}^{-}$from entering thyroid follicular cells and interfering with the key steps of thyroid hormone biosynthesis [17]. However, the results have been reported on the effects of perchlorate and iodine on thyroid hormone levels are inconsistent. The effects of perchlorate on human thyroid function appear to be related to exposure dose, physiological feedback regulation mechanism, the combined effects of multiple endocrine disruptors, and nutritional iodine status, and so on [18-20]. Furthermore, there are limited reports showing that perchlorate exposure may increase the risk 
of thyroid papillary carcinoma (PTC) [21]. However, the relationships between perchlorate, iodine, and different pathological types of human thyroid tumors have not yet been reported.

Therefore, exploring the effects of perchlorate and iodine on three different pathological types of thyroid tumors and on thyroid function is urgently needed. The purpose of the present study was to investigate the effect of perchlorate and iodine on the risk of nodular goiter (NG), papillary thyroid microcarcinoma (PTMC), and PTC and to investigate the correlation with thyroid function indicators, so as to identify the key risk factors for thyroid disease. The study will provide a scientific basis for the prevention and control of thyroid tumors in future.

\section{Methods}

\section{Study population}

This study recruited 184 patients with thyroid tumors and NG and 211 controls among total 395 participants, of which 184 case-control pairs were matched by gender and age ( \pm 2 years). The patients with newly diagnosed thyroid tumors and nodular goiter, not undergoing radiotherapy and chemotherapy, from September 2018 to September 2019 were recruited at a specialist clinic from two comprehensive third class hospitals in Shenzhen. They included 72 patients with NG, 46 patients with PTMC and 66 patients with PTC. All the three pathological types categorized as total cases. These patients met the "NCCN Clinical Practice Guidelines in Oncology: Thyroid Carcinoma" diagnostic guidelines issued by the National Comprehensive Cancer Network (NCCN) [22]. All participants had lived in Shenzhen for more than 6 months and signed the informed consent form. They were invited to participate in the face-to-face questionnaire survey. Blood and urine samples were taken from the participants. The control population was tested for five thyroid hormone indicators, two antibodies, and B-ultrasound, for those with normal thyroid function and ultrasound were defined as the control group. Exclusion criteria for the cases and controls were as follows: severe heart, liver, and kidney diseases; previous history of thyroid disease; use of iodine, thyroid hormones, and other drugs; pregnancy or recent use of contraceptives or estrogen; terminal cancer; use of radiotherapy and chemotherapy. The present study was approved by the Ethics Committee of the Shenzhen Center for Disease Control and Prevention and the two recruiting hospitals ([2019]012A).

\section{Sample collection and detection methods}

Fasting venous blood was collected from the participants, and serum was separated by centrifugation at $3000 \mathrm{rpm}$ for $15 \mathrm{~min}$ within $2 \mathrm{~h}$ and stored in cryogenic vials. The morning fasting middle-segment urine was collected and transferred into centrifuge tubes for storage. All samples were stored at $-80^{\circ} \mathrm{C}$ in an ultra-low temperature refrigerator until analysis.

Serum levels of thyroid function indicators, including thyroid stimulating hormone (TSH), triiodothyronine (TT3), thyroxine (TT4), free triiodothyronine (FT3), free thyroxine (FT4), thyroid peroxidase antibody (TPOAb), and thyroglobulin antibody (TgAb), were measured by an automatic chemiluminescence analyzer (Maglumi 2000, Shenzhen New Industries Biomedical Engineering Co., Ltd., China). The reference range appears below: TSH: 0.30-4.50 $\mu \mathrm{IU} / \mathrm{mL}$; TT3: $0.69-2.15 \mathrm{ng} / \mathrm{mL}$; TT4: 52.00 $127.00 \mathrm{ng} / \mathrm{mL}$; FT3: $2.00-4.20 \mathrm{pg} / \mathrm{mL}$; FT4: $8.90-17.20 \mathrm{\rho g} /$ $\mathrm{mL}$; TgAb: $0.00-95.00 \mathrm{IU} / \mathrm{mL}$; TPOAb: $0.00-30 \mathrm{IU} / \mathrm{mL}$ [22].

Perchlorate in urine was detected by high-performance liquid chromatography with tandem mass spectrometry (HPLC-MS/MS). Specifically, $570 \mu \mathrm{L}$ of urine sample was added into a $2-\mathrm{mL}$ centrifuge tube; $30 \mu \mathrm{L}$ of $1 \mu \mathrm{g} / \mathrm{mL}$ perchlorate isotope internal standard was added, mixed, and passed through $0.2-\mu \mathrm{m}$ filter membrane. Finally, $5 \mu \mathrm{L}$ of the solution was injected into the Infinity Lab Poroshell 120 HILIC-Z column $(2.1 \times 100 \mathrm{~mm} \times 2.7 \mu \mathrm{m}$; Agilent Technologies, CA, USA). The standard substance of perchlorate was purchased from Beijing North Weiye Institute of Metrology and Technology (Beijing, China), and the internal standard substance was purchased from Shanghai ZZBIO Co. Ltd. (Shanghai, China). Two quality control samples were detected for every 20 samples, and the spiked recovery rate for samples was verified according to the low, medium, and high concentration of the samples. The limits of detection and quantification were 0.083 and $0.275 \mu \mathrm{g} / \mathrm{L}$, respectively, and the spiked standard recovery was found between 93.2 and $115.5 \%$.

The level of iodine in urine was evaluated by inductively coupled plasma mass spectrometry (ICP-MS, Agilent $7700 \mathrm{x}$, Agilent Technologies, USA). First, $1 \mathrm{~mL}$ of urine sample was accurately added into a $15-\mathrm{mL}$ centrifuge tube, diluted to $10.0 \mathrm{~mL}$ with $0.25 \%$ tetramethylammonium hydroxide solution, oscillated on the vortex mixer, and then injected onto the ICP-MS. The standard substance for the analysis of iodine composition in freeze-dried human urine (purchased from the National Reference Laboratory for iodine deficiency disorders, Beijing, China) was used as the quality control sample to control the whole process of detection.

The levels of perchlorate and iodine in urine were corrected by urinary specific gravity (USG), and the formula is as follows [23] $\left(C_{c}\right.$ : corrected concentration; $C_{d}$ : actual detected concentration; USG $_{m}$ : the median of USG in urine of case or control group; $\mathrm{USG}_{d}$ : actual detected USG in individual urine): 


$$
C_{c}=C_{d} \times \frac{U S G_{m}-1}{U S G_{d}-1}
$$

\section{Statistical analyses}

SPSS Statistics 25.0 (IBM, Chicago, USA) and R 3.6.3 (Lucent Technologies, USA) were used for statistical analysis of the data. Categorical variables were compared by the chi-square test. Mean \pm SD (standard deviation) or median (interquartile range) was used to describe continuous variables. The Wilcoxon signed-rank test was used for intergroup comparisons, and the Kruskal-Wallis test was used for comparison of multiple groups.

Conditional logistic regression was used to assess the risk of thyroid tumors and NG related to urinary target variables (perchlorate, iodine) by using crude and adjusted odds ratios (ORs) and their corresponding 95\% confidence intervals (CIs). According to WHO standards, iodine nutrition levels were divided into insufficient $(<100 \mu \mathrm{g} / \mathrm{L})$, adequate $(100-200 \mu \mathrm{g} / \mathrm{L})$, more than adequate $(200-300 \mu \mathrm{g} / \mathrm{L})$, and excess $(\geq 300 \mu \mathrm{g} / \mathrm{L})$. Multiple conditional logistic regression analyses with $P \leq 0.10$ as the inclusion criterion were conducted, and body mass index (BMI), smoking, and drinking were included as confounding variables. Restricted cubic spline regression with three knots (10th, 50th, and 90th percentiles) was used to evaluate the dose-response relationship between perchlorate exposure and the risk of thyroid tumors and NG. The relationship between perchlorate, iodine, and thyroid function indicators was analyzed only in the controls. Spearman's rank correlation and multiple linear regression analysis were used to evaluate the relationship between urine target variables (perchlorate and iodine) and TSH, TT3, TT4, FT3, FT4, TPOAb, and TgAb in the control group. The variables with non-normal distribution (perchlorate, iodine, TSH, TPOAb, and TgAb) were natural log-transformed. Multiple linear regression model adjusted for gender, age, BMI, smoking, and drinking was established. All statistical significance level was set at $P<0.05$ (two-sided).

\section{Results}

Population characteristics are presented in Table 1. Among the 184 pairs of cases and controls, men and women accounted for 35.3 and $64.7 \%$, respectively, with the female-to-male ratio of 1.8:1. The majority of patients were in the youth group ( $\leq 44$ years old), followed by the middle-aged group ( $45-59$ years old) and

Table 1 The characteristics of study population

\begin{tabular}{|c|c|c|c|}
\hline Characteristics & Controls $(n=184)$ & Cases $(n=184)$ & $P$-value \\
\hline Gender, n (\%) & & & - \\
\hline Male & $65(35.3)$ & $65(35.3)$ & \\
\hline Female & $119(64.7)$ & $119(64.7)$ & \\
\hline Age (years), n (\%) & & & - \\
\hline$\leq 44$ & $115(62.5)$ & $116(63.0)$ & \\
\hline $45-59$ & $51(27.7)$ & $49(26.6)$ & \\
\hline$\geq 60$ & $18(9.8)$ & $19(10.4)$ & \\
\hline BMI (kg/m², Mean \pm SD) & $23.37 \pm 3.45$ & $22.23 \pm 2.74$ & $<0.001$ \\
\hline Educational level, n (\%) & & & 0.746 \\
\hline Elementary school and below & $18(9.8)$ & $23(12.5)$ & \\
\hline Junior/ high school & $88(47.8)$ & $80(43.5)$ & \\
\hline College Degree and above & $78(42.4)$ & $81(44.0)$ & \\
\hline Duration of residence (years), n (\%) & & & 0.219 \\
\hline$\leq 5$ & $19(10.3)$ & $22(12.0)$ & \\
\hline $5-10$ & $53(28.8)$ & $38(20.7)$ & \\
\hline$>10$ & $112(60.9)$ & $124(67.4)$ & \\
\hline Seafood, n (\%) & & & 0.325 \\
\hline$<1$ times/week & $27(14.7)$ & $31(16.8)$ & \\
\hline $1-2$ times/week & $139(75.5)$ & $144(78.3)$ & \\
\hline$>2$ times/week & $18(9.8)$ & $9(4.9)$ & \\
\hline Smoking, n (\%) & $27(14.7)$ & $37(20.1)$ & 0.134 \\
\hline Drinking, n (\%) & $69(37.5)$ & $97(52.7)$ & 0.002 \\
\hline
\end{tabular}

Note: $B M I$ Body mass index, SD Standard deviation

$P$-value were derived from chi-square tests for categorical variables, Wilcoxon signed-rank test for continuous variables 
the elderly group ( $\geq 60$ years old), accounting for 63.0 , 26.6 , and $10.4 \%$, respectively. In the univariate analyses, the case group had a lower BMI and a higher proportion of individuals with drinking habit than the control group $(P<0.05)$.

The concentration distribution of perchlorate and iodine in urine is shown in Table 2. Perchlorate concentrations were higher in total cases compared to total controls $(P<0.05)$. After pathological classification, perchlorate concentrations were higher in NG and PTC than in the corresponding controls $(P<0.05)$. There was no significant difference among the three pathological types $(P>0.05)$. Furthermore, the levels of iodine in urine were not significantly different between total cases, NG, PTMC, PTC, and their corresponding controls $(P>0.05)$.

Furthermore, potential confounding variables for thyroid tumors and nodular goiter have been evaluated (Supplementary Material, Table 1), and BMI, smoking, and drinking were included as confounding variables in multiple conditional logistic regression analyses (Table 3$)$. The results showed that perchlorate was positively associated with the risk of total cases $(\mathrm{OR}=1.041$,
95\% CI: 1.017, 1.066) after adjusting for covariates. The association between perchlorate and the different thyroid pathological types was further analyzed, and positive association with the risk of PTC was found $(\mathrm{OR}=1.058$, 95\% CI: $1.009,1.110)$, while no significant association was found between perchlorate and NG and between perchlorate and PTMC $(P>0.05)$. Moreover, there was no significant association between iodine and thyroid tumors and NG $(P>0.05)$.

Restricted cubic spline regression analysis showed a non-linear dose-response relationship between perchlorate and PTC (P-non-linear <0.05) (Fig. $1 \mathrm{D}$ and Supplementary Material, Table 2). The risk of PTC did not appear until perchlorate concentration reached $11.33 \mu \mathrm{g} / \mathrm{L}$, and the ORs showed an increasing trend (1.008-1.924) for perchlorate concentrations between 11.33 and $27.04 \mu \mathrm{g} / \mathrm{L}$. However, the risk trend for perchlorate on PTC was relatively flat and not monotonic, with the risk association disappearing when perchlorate concentrations was over $27.04 \mu \mathrm{g} / \mathrm{L}$. The dose-response relationship between perchlorate and total cases and between perchlorate and NG was significant in the

Table 2 Differences of perchlorate, iodine levels in urine between control and case group

\begin{tabular}{|c|c|c|c|c|c|c|c|c|c|}
\hline \multirow[t]{2}{*}{ Variables } & \multirow{2}{*}{$\begin{array}{l}\text { Pathological } \\
\text { type }\end{array}$} & \multicolumn{2}{|c|}{ Overall participants } & \multicolumn{2}{|l|}{ Controls } & \multicolumn{2}{|l|}{ Cases } & \multirow[t]{2}{*}{$P$-value ${ }^{\text {a }}$} & \multirow[t]{2}{*}{$P$-value ${ }^{b}$} \\
\hline & & Median & $\begin{array}{l}\text { 25th-75th } \\
\text { percentiles }\end{array}$ & Median & $\begin{array}{l}\text { 25th-75th } \\
\text { percentiles }\end{array}$ & Median & $\begin{array}{l}\text { 25th-75th } \\
\text { percentiles }\end{array}$ & & \\
\hline \multirow[t]{5}{*}{ Perchlorate $(\mu \mathrm{g} / \mathrm{L})$} & & 11.27 & $6.89-18.49$ & & & & & & \\
\hline & Total cases & & & 9.29 & $6.35-14.74$ & 14.31 & $8.25-20.58$ & $<0.001$ & - \\
\hline & NG & & & 9.68 & $6.48-15.22$ & 14.56 & $7.88-22.18$ & 0.037 & 0.998 \\
\hline & PTMC & & & 9.95 & $6.88-14.70$ & 14.13 & $7.56-21.64$ & 0.061 & \\
\hline & PTC & & & 8.73 & $5.75-13.39$ & 14.27 & 8.67-19.16 & 0.002 & \\
\hline \multirow[t]{5}{*}{ lodine $(\mu \mathrm{g} / \mathrm{L})$} & & 131.23 & $84.00-197.84$ & & & & & & \\
\hline & Total cases & & & 131.59 & 82.13-183.25 & 131.23 & $84.46-214.92$ & 0.153 & - \\
\hline & NG & & & 122.67 & $66.63-171.41$ & 130.19 & $79.60-205.02$ & 0.165 & 0.836 \\
\hline & PTMC & & & 138.58 & 100.43-181.96 & 148.34 & $95.15-213.52$ & 0.589 & \\
\hline & PTC & & & 130.35 & $84.74-189.80$ & 126.97 & $88.82-215.71$ & 0.697 & \\
\hline
\end{tabular}

Note: Data are presented as medians and 25-75 percentiles. Perchlorate and iodine were corrected by urinary specific gravity (USG). NG nodular goiter, PTMC papillary thyroid micro carcinoma, PTC papillary thyroid carcinoma, Total cases including NG, PTMC and PTC

a $P$-value were derived from Wilcoxon signed-rank test between the case and control group

b $P$-value was derived from the Kruskal-Wallis test between the three types of pathological

Table 3 The association between perchlorate, iodine and thyroid tumors and nodular goiter by conditional logistic model

\begin{tabular}{|c|c|c|c|c|c|c|c|c|}
\hline \multirow[t]{2}{*}{ Variables } & \multicolumn{2}{|l|}{ Total cases } & \multicolumn{2}{|l|}{ NG } & \multicolumn{2}{|l|}{ PTMC } & \multicolumn{2}{|l|}{ PTC } \\
\hline & ORs (\%95CI) & $P$-value & ORs (\%95CI) & $P$-value & ORs (\%95CI) & $P$-value & ORs (\%95CI) & $P$-value \\
\hline N (case/control) & $184 / 184$ & & $72 / 72$ & & $46 / 46$ & & $66 / 66$ & \\
\hline Perchlorate & $1.041(1.017,1.066)$ & 0.001 & $1.034(0.994,1.074)$ & 0.094 & $1.033(0.996,1.072)$ & 0.084 & $1.058(1.009,1.110)$ & 0.021 \\
\hline lodine & $1.000(1.000,1.000)$ & 0.759 & $1.002(0.998,1.005)$ & 0.303 & $1.000(0.998,1.001)$ & 0.596 & $1.000(0.999,1.002)$ & 0.518 \\
\hline
\end{tabular}

Note: Perchlorate and iodine were corrected by urinary specific gravity (USG). Perchlorate, iodine, BMI, smoking, and drinking were included in the model. OR odds ratio, Cl confidence interval, NG nodular goiter, PTMC papillary thyroid micro carcinoma, PTC papillary thyroid carcinoma, Total cases including NG, PTMC and PTC 
A

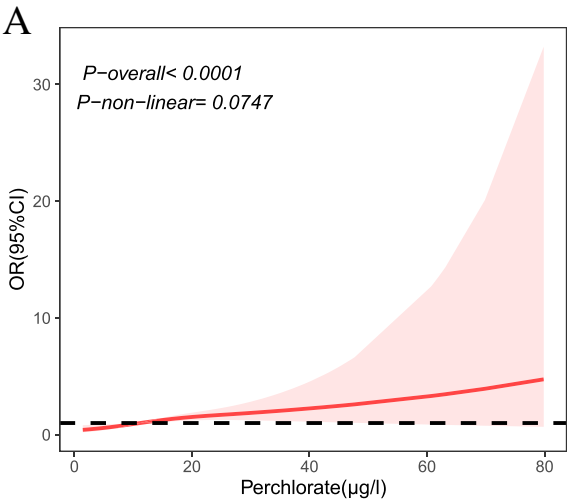

C

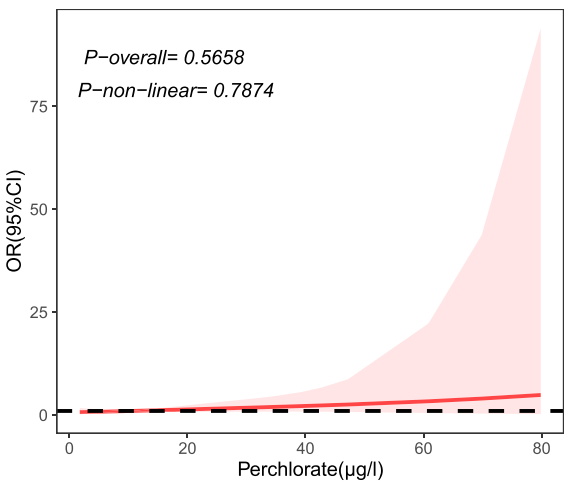

B

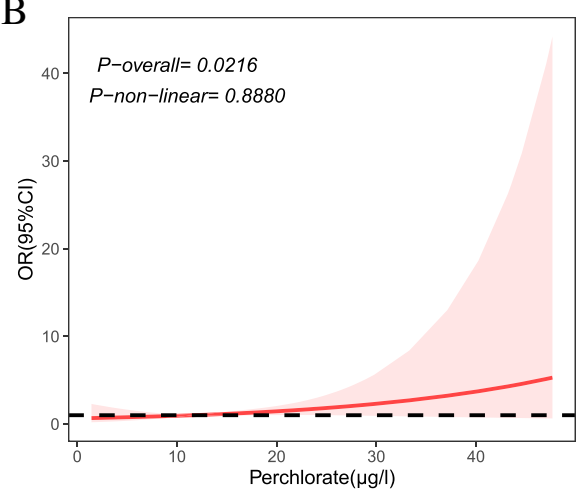

$\mathrm{D}$

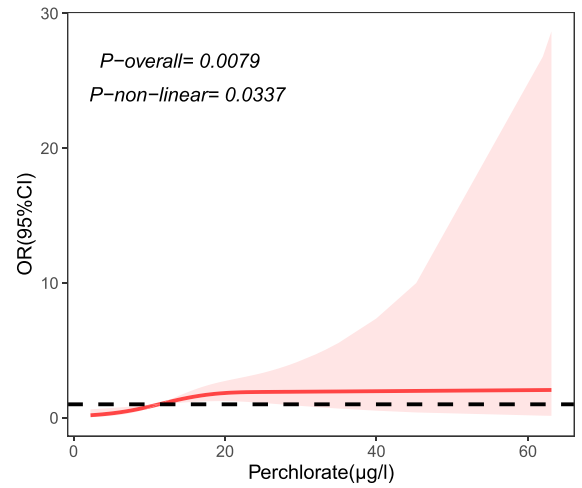

Fig. 1 Dose-response relationship between perchlorate exposure and thyroid tumor and NG by restricted cubic spline (RCS). Perchlorate was corrected by urinary specific gravity (USG). A for total cases, B for NG, C for PTMC, D for PTC; estimates adjusted for BMI, smoking, drinking, and iodine; the red line represented the calculated ORs, the dotted line represented the baseline of $\mathrm{OR}=1$, the shades of red represent the $95 \% \mathrm{Cl}$

overall eq. $(P$-overall $<0.05)$ but also belonged to a nonmonotonic trend (Fig. $1 \mathrm{~A}$ and B). There was no statistically significant dose-response relationship between perchlorate and PTMC (Fig. $1 \mathrm{C}$ ).

Correlations between the thyroid function indicators and the levels of perchlorate and iodine were further analyzed in the control group. After adjusted for gender, age, BMI, smoking, and drinking, perchlorate correlated negatively with FT3 and positively with TgAb, with a $\beta$ value of -0.067 (95\% CI: $-0.12,-0.013)$ and 0.15 (95\% CI: $0.0053,0.30$ ), respectively (Table 4). No significant effect of iodine on thyroid function indicators was observed $(P>0.05)$ (Table 4$)$. Next, the effect of perchlorate on thyroid function indicators was further

Table 4 Multiple linear regression between perchlorate, iodine and thyroid function indicators in the control group

\begin{tabular}{lllll}
\hline Indicators & Perchlorate & & lodine \\
\cline { 2 - 3 } & $\boldsymbol{\beta}$ (\%95Cl) & $\boldsymbol{P}$-value & $\boldsymbol{\beta}$ (\%95Cl) & $\boldsymbol{P}$-value \\
\hline TSH & $0.045(-0.043,0.13)$ & 0.322 & $-0.021(-0.067,0.025)$ & 0.370 \\
TT3 & $0.0018(-0.037,0.040)$ & 0.929 & $-0.0083(-0.029,0.012)$ & 0.422 \\
TT4 & $1.74(-0.82,4.29)$ & 0.185 & $-0.37(-1.71,0.97)$ & 0.588 \\
FT3 & $-0.067(-0.12,-0.013)$ & 0.016 & $-0.0043(-0.033,0.024)$ & 0.768 \\
FT4 & $0.018(-0.0048,0.040)$ & 0.125 & $0.0067(-0.0051,0.019)$ & 0.268 \\
TPOAb & $0.014(-0.20,0.23)$ & 0.900 & $-0.0077(-0.12,0.11)$ & 0.895 \\
TgAb & $0.15(0.0053,0.30)$ & 0.044 & $-0.0075(-0.085,0.071)$ & 0.851 \\
\hline
\end{tabular}

Note: Perchlorate and iodine were corrected by urinary specific gravity (USG); Perchlorate, iodine, gender, age, BMI, smoking, and drinking were included in the model. Perchlorate, iodine, TSH, TPOAb and TgAb were natural logarithm conversion. $\mathrm{Cl}$ confidence interval, TSH thyroid stimulating hormone, TT3 triiodothyronine; TT4 thyroxine, FT3 free triiodothyronine, FT4 free thyroxine, TPOAb thyroid peroxidase antibody, TgAb thyroglobulin antibody 
evaluated at three levels of iodine: insufficient, adequate, and more than adequate or excess (Table 5). The results showed that perchlorate positively correlated with TSH at iodine adequate levels, with a $\beta$ value of 0.19 (95\% CI: $0.040,0.33)$. When the iodine level was at more than adequate or excess, perchlorate correlated negatively with FT3 and positively with $\mathrm{TgAb}$, with a $\beta$ value of -0.12 (95\% CI: $-0.23,-0.0049)$ and 0.37 (95\% CI: 0.023, 0.71), respectively.

\section{Discussion}

The present study demonstrated a positive association between perchlorate and the risk of PTC and perchlorate can disturb the homeostasis of thyroid function, but did not find an association between perchlorate and NG and PTMC. In addition, no association was found between iodine and the risk of thyroid tumors and nodular goiter, and thyroid function. The association of endocrine-disrupting compounds and iodide inhibitors with thyroid

Table 5 Correlation between perchlorate and thyroid function under different iodine levels

\begin{tabular}{|c|c|c|c|}
\hline Indicators & $\beta$ & $\% 95 \mathrm{Cl}$ for $\beta$ & $P$-value \\
\hline \multicolumn{4}{|c|}{ With inadequate iodine levels $(n=53)$} \\
\hline TSH & 0.020 & $(-0.13,0.17)$ & 0.799 \\
\hline TT3 & 0.013 & $(-0.059,0.086)$ & 0.721 \\
\hline TT4 & 3.80 & $(-0.87,8.47)$ & 0.118 \\
\hline FT3 & -0.041 & $(-0.14,0.059)$ & 0.429 \\
\hline FT4 & 0.020 & $(-0.022,0.062)$ & 0.358 \\
\hline TPOAb & 0.41 & $(-0.037,0.86)$ & 0.079 \\
\hline $\operatorname{TgAb}$ & 0.14 & $(-0.08,0.35)$ & 0.226 \\
\hline \multicolumn{4}{|c|}{ With adequate iodine levels $(n=87)$} \\
\hline TSH & 0.19 & $(0.040,0.33)$ & 0.015 \\
\hline TT3 & -0.013 & $(-0.082,0.056)$ & 0.715 \\
\hline TT4 & 2.46 & $(-2.00,6.92)$ & 0.283 \\
\hline FT3 & -0.091 & $(-0.19,0.0044)$ & 0.065 \\
\hline FT4 & 0.011 & $(-0.032,0.055)$ & 0.609 \\
\hline TPOAb & -0.18 & $(-0.55,0.19)$ & 0.349 \\
\hline $\operatorname{TgAb}$ & 0.021 & $(-0.25,0.29)$ & 0.883 \\
\hline \multicolumn{4}{|c|}{ With more than adequate or excess iodine levels $(n=71)$} \\
\hline TSH & 0.029 & $(-0.17,0.23)$ & 0.774 \\
\hline TT3 & -0.071 & $(-0.14,0.0021)$ & 0.061 \\
\hline TT4 & -2.55 & $(-7.88,2.79)$ & 0.353 \\
\hline FT3 & -0.12 & $(-0.23,-0.0049)$ & 0.045 \\
\hline FT4 & 0.025 & $(-0.015,0.066)$ & 0.224 \\
\hline TPOAb & 0.10 & $(-0.31,0.51)$ & 0.620 \\
\hline $\operatorname{Tg} A b$ & 0.37 & $(0.023,0.71)$ & 0.041 \\
\hline
\end{tabular}

Note: Perchlorate and iodine were corrected by urinary specific gravity (USG); Perchlorate, iodine, gender, age, BMI, smoking, and drinking were included in the model. Perchlorate, iodine, TSH, TPOAb and TgAb were natural logarithm conversion. Cl confidence interval, TSH thyroid stimulating hormone, TT3 triiodothyronine; TT4 thyroxine, FT3 free triiodothyronine, FT4 free thyroxine, $T P O A b$ thyroid peroxidase antibody, $\operatorname{TgAb}$ thyroglobulin antibody diseases has received worldwide attention in recent years. However, few population-based studies have examined the relationship between iodination inhibitors and thyroid diseases. To our knowledge, the present study is the first population-based study to simultaneously examine the association of perchlorate exposure with three thyroid neoplasma types (NG, PTMC, and PTC).

Our results showed a positive association between perchlorate exposure and the risk of PTC $(\mathrm{OR}=1.058,95 \%$ CI: $1.009,1.110)$ with non-linear dose-response relationship $(P$-non-linear $<0.05)$. This is consistent with another small population study conducted in China, in which the association of perchlorate exposure and PTC was found $(\mathrm{OR}=2.27,95 \% \mathrm{CI}: 1.03-5.03)$ [21]. The risk of perchlorate on PTC that we found was marginal and the effect of small sample size needs to be considered. In addition, humans are generally exposed to multiple endocrine disruptors in the external environment simultaneously [8], thus it is worth considering whether perchlorate act synergistically or antagonistically when combined with them on the thyroid. For example, Zhang et al., 2018 [21] included both perchlorate and thiocyanate and obtained a higher OR for the risk association than our study $(2.27>1.058)$, so the combined effect of other thyroid disruptors on thyroid tumors cannot be excluded. It is worth noting that the initial concentration of perchlorate associated with the PTC risk was between the median value and the $P_{75}$ level in the controls from the present study, which provided a statistical reference for the human exposure threshold. Animals studies have shown that exposure to perchlorate increases the size and number of thyroid follicles, which leads to structural changes in the thyroid gland [24]. However, previous studies have not determined whether perchlorate is involved in the initiating or the promoting mechanism in thyroid tumors. In the present study, we found a positive association between perchlorate and NG in univariate model, but this association disappeared in multifactorial model. It is possible that perchlorate is indeed not associated with NG, or the association may have been masked by some confounding factors not taken into account in our study. A report on perchlorate drinking water exposure test in rats from 2 weeks prior to cohabitation to lactation day 10 found that thyroid weight increased in female pups at $1.0 \mathrm{mg} / \mathrm{kg} / \mathrm{d}$ ammonium perchlorate (AP) exposure compared to controls on day 22 of lactation [25]. Moreover, increased thyroid weight and decreased colloid in female rats and male $/$ female pups were observed at $30.0 \mathrm{mg} / \mathrm{kg} / \mathrm{d}$ AP exposure, further follicular hypertrophy was induced in female rats, suggesting that perchlorate may impair thyroid cells and alter thyroid morphology with gender sensitivity [25]. NG is also an irreversible pathological change that can be transformed from a diffuse goiter, 
thus the association of perchlorate with NG can be further investigated. In addition, no statistical association of perchlorate with the risk of PTMC was found either. Compared with PTC, PTMC is clinically asymptomatic, smaller in diameter $(<10 \mathrm{~mm})$, and has a much lower mortality rate [26]. Given that we found that perchlorate exposure was associated with the risk of PTC but not PTMC, perchlorate exposure likely influences tumor size and probably acts as a promoter of TC rather than an initiator.

Furthermore, BMI was found to be a protective factor for thyroid tumors and NG, while drinking was a risk factor in this study. Similar findings were reported in Mijović T et al., 2009 [27] and 2010 to 2011 Korean National Health and Nutrition Examination Survey (KNHANES) [28]. However, recent studies show that BMI is positively correlated with papillary and follicular carcinomas of the thyroid, and this may be related to elevate TSH induced by obesity, chronic inflammatory response and oxidative stress, of which elevated TSH is considered a risk factor for TC [29]. Moreover, the study by Yeo et al. showed that smoking and drinking were negatively correlate with TC, which may be related to the lowering of TSH induced by smoking and drinking, as well as the anti-estrogenic effects [30]. These above findings suggest that confounding factors such as BMI, smoking, and drinking may affect the development of thyroid tumors and have been controlled in the following multivariate analysis of this study.

The effects of perchlorate on the indicators of thyroid function were assessed in this study, and the results showed that perchlorate was negatively correlated with FT3 and positively correlated with $\mathrm{TgAb}$, and only positively correlated with TSH when iodine at adequate levels. According to the National Health and Nutrition Examination Survey (NHANES) in the United States, perchlorate was positively associated with TSH only in women aged above 12 years, regardless of whether iodine was adequate or not; in contrast, perchlorate was negatively associated with TT4 only in iodine deficiency status, suggesting an association between perchlorate and hypothyroidism [18]. However, perchlorate has been found to be positively associated with TSH in pregnant women only when co-exposed with thiocyanate and nitrate rather than independently [31]. A subchronic toxicity test study in rats showed that AP exposure was negatively associated with TT3 and TT4 and positively associated with $\mathrm{TSH}$, which occurred only at doses of up to $10 \mathrm{mg} / \mathrm{kg} / \mathrm{day}$ of AP exposure [32]. However, no significant changes in thyroid function were found in both trials from 9 healthy male volunteers for 2-week exposure at perchlorate dose $10 \mathrm{mg} / \mathrm{d}$ and 13 healthy volunteers for 6-month at exposure levels of $3 \mathrm{mg} / \mathrm{d}$ and $0.5 \mathrm{mg} / \mathrm{d}$, and the small sample size for the above two studies may influence the statistical power. Nevertheless, effects of high-dose perchlorate exposure or lower doses of more prolonged perchlorate exposure on thyroid function haven't been demonstrated in the general population till now [33, 34]. This indicates that perchlorate interference with TSH is also influenced by gender, exposure dose, and combined effects with other NIS inhibitors. We found the negative correlation of perchlorate and FT3 but not the positive correlation between perchlorate and TSH at the same iodine level, which may be related to sample size limitation or other confounding factors. In fact, it has been reported that TSH can stimulate the transcription and biosynthesis of NIS [17]. The above results might suggest the following possible mechanism: perchlorate competes with iodine for NIS to reduce iodine uptake in the thyroid gland, thereby inducing a decrease in FT3, which can reflect TT3 or TT4 decrease when not accompanied by thyroid hormone binding protein decrease, and increasing the secretion of TSH under the regulation of a negative feedback mechanism, while TSH restores the iodide level in thyroid cells by upregulation of NIS. In addition, the association of thyroid hormones and thyroid autoimmune antibodies with the risk of thyroid tumors has been reported $[35,36]$. It has been shown that TSH can activate the phospholipase C/protein kinase C (PLC-PKC) signaling pathway to stimulate the secretion of vascular endothelial growth factor (VEGF) and induce neoangiogenesis, thereby promoting the proliferation of TC cells [37], which suggests that elevated TSH due to perchlorate exposure may have a role in promoting TC.

Both TPOAb and TgAb are markers of thyroid autoimmunity and can assist in the prediction of thyroid disease, such as hypothyroidism and prognosis of hyperthyroidism treatment [38]. Our study found a positive correlation between perchlorate and $\mathrm{TgAb}$, suggesting that perchlorate may be associated with thyroid cell damage. However, since autoimmune thyroid disease usually requires a combination of positive $\mathrm{Tg} \mathrm{Ab}$ and $\mathrm{TPOAb}$ for diagnosis, the effect of perchlorate on thyroid autoimmunity needs to be further verified. In particular, TgAb can also be used as a surveillance indicator for differentiated thyroid cancer (TDC) and has important implications for predicting the prognosis of TDC.

For iodine, we found no association with the risk of thyroid tumors and NG. Wang et al., 2014 pointed out that high iodine levels significantly correlate with the occurrence of benign and malignant thyroid tumors and the aggressiveness of PTC [39]. However, the median urinary iodine concentration in their study was 331.33 and $466.23 \mu \mathrm{g} / \mathrm{L}$ for thyroid nodules and TC, among which the iodine excess accounted for 62.75 and $66.99 \%$, respectively, and much higher than that in our study [39]. In the 
present study, the median urinary iodine concentration was $130.19,148.34$ and $126.97 \mu \mathrm{g} / \mathrm{L}$ for NG, PTMC and PTC, respectively, while only 12.50 and $7.14 \%$ of patients with NG and TC (including PTMC and PTC) were at iodine excess levels (Supplementary Material, Table 4). Thus, the result of correlation between iodine and thyroid tumor from high iodine levels at iodine excess was no comparability with the present study. Several previous studies have demonstrated that the relationship between iodine and thyroid tumors is more evident at ultralow and ultra-high intakes. For instance, experimental research revealed that ultra-low $\left(1.0 \times 10^{-6} \mathrm{~mol} / \mathrm{L}\right)$ and ultra-high dose $\left(1.0 \times 10^{-3} \mathrm{~mol} / \mathrm{L}\right)$ of iodine induced high expression of the SPANXA1 gene and promoted TC cell proliferation and apoptosis [40]. Furthermore, metaanalyses of animal and human studies have shown that both chronic iodine insufficiency and excess were risk factors for TC, but iodine intake was not a significant independent factor in the development of thyroid disease [41]. This is consistent with two epidemiological studies suggesting that high iodine intake may play a role in the tumor size and capsular invasion compared with the occurrence of PTC $[42,43]$.

Iodine plays an important role in the synthesis of thyroid hormones. However, no correlation between iodine levels and the thyroid function indicators was found in this study. This is similar to a previous cross-sectional study in China, which found no significant association between iodine levels and TSH, FT3, or FT4 [44]. Similarly, a large population study of over 7000 participants (NHANES) found no correlation between iodine levels and TSH and TT4 [45]. In contrast, studies based on complete food frequency surveys to assess iodine levels among young people residing in the mountains of the western United States have shown that iodine levels positively correlate with TSH [46]. The great variation among the studies may reflect the physiological regulation of normal human thyroid, where thyroid hormones are not significantly affected unless the thyroid gland is overloaded [47]. In addition, Wang et al., 2019 showed that both iodine deficiency and iodine excess are risk factors for autoimmune thyroid diseases [48]. Although both $\mathrm{TgAb}$ and TPOAb levels in the present study increased with the increase in iodine nutrition level, their variance at different iodine nutrition levels was not statistically significant and the antibody levels were still within the normal range (Supplementary Material, Table 5). It has been suggested that iodine is more likely to induce thyroid autoimmunity through unmasking a cryptic epitope on thyroglobulin [49]. The results of above study were only obtained from a single measurement. In fact, as individual iodine levels are closely related to the diurnal variation of dietary iodine content [50], it is even more important to observe fluctuations in thyroid hormones in the population by monitoring iodine nutrition levels over time.

The source of perchlorate exposure for the recruited population was analyzed in this study. The median perchlorate concentration in this study $(11.27 \mu \mathrm{g} / \mathrm{L})$ was notably higher than that of 6044 participants of the general population included in the NHANES $(3.9 \mu \mathrm{g} / \mathrm{L})$ [19]; however, the US study included not only multiregional and multiethnic populations but also adolescent population, suggesting that demographic and geographical variations may contribute to the differences in perchlorate exposure. Human perchlorate exposure has been related to the living environment and dietary intake. Studies of people living in fireworks production sites have shown that the mean concentration of perchlorate in urine is 16 times higher than that in the present study [9]. Research has supported that food intake contributes $86 \%$ to perchlorate exposure [51]. Although there have been no reports of perchlorate contamination in Shenzhen foods, limited studies have shown that perchlorate residues were found in vegetables, fruits, grains, and dairy products, especially in leafy vegetables (spinach) [10]. Moreover, the study of perchlorate concentration in bottled water and groundwater from 15 cities in China showed that the highest concentration $(54.4 \mu \mathrm{g} / \mathrm{L})$ was detected in Hengyang, Hunan Province-a fireworks production site, while Shenzhen was at the lowest concentration $(0.14 \mu \mathrm{g} / \mathrm{L})$ [13], suggesting that drinking water may not be the primary source of perchlorate exposure of the recruited population. Thus, the main source of perchlorate exposure in Shenzhen residents was more likely to be food intake. Further investigation on perchlorate exposure for Shenzhen residents is needed in the future.

There are some strengths and limitations in this study. First, cases with three pathological types of thyroid tumor and corresponding controls were matched by gender and age and recruited based on strict inclusion and exclusion criteria. Second, the authenticity and accuracy of data were ensured under strict quality control measures. The confounding variables were adjusted for using a multifactorial model. This study was the first to compare the effects of perchlorate exposure on the three types of thyroid neoplasms. Thus, the findings provide important etiological evidence for thyroid tumor and nodular goiter in order to improve thyroid disease prevention in future. However, there are some limitations to this study. First, the sample size in different pathological types was small, especially in the PTMC group. Second, the levels of perchlorate and iodine in urine were based on a single measurement as it is difficult to reflect long-term human burden levels in a cross-sectional study. However, the present study found the association of perchlorate with 
PTC and first indicated that perchlorate has no statistical association with the risk of NG and PTMC. In future, population-based cohort studies with precisely monitored iodine intake through long-term follow-up in the exposed populations from perchlorate-contaminated areas should be conducted.

\section{Conclusions}

We found that perchlorate can increase the risk of PTC in a non-linear dose-response relationship, but no significant associations with NG and PTMC. Moreover, perchlorate can disturb thyroid hormone homeostasis and thyroid autoantibody levels. However, the association between iodine and thyroid tumors, NG, and thyroid function indicators has not been found in this study. The present study provided important population evidence for the identification of the risk factors of thyroid tumors and NG from the exogenous environment chemical exposure.

\section{Abbreviations}

BMl: Body mass index; OR: Odds ratio; Cl: Confidence interval; NG: Nodular goiter; PTMC: Papillary thyroid microcarcinoma; PTC: Papillary thyroid carcinoma; TC: Thyroid cancer; TNs: Thyroid nodules; TSH: Thyroid stimulating hormone; TT3: Triiodothyronine; TT4: Thyroxine; FT3: Free triiodothyronine; FT4: Free thyroxine; TPOAb: Thyroid peroxidase antibody; TgAb: Thyroglobulin antibody; USG: Urinary specific gravity; NIS: Sodium/lodide Symporter; AP: Ammonium perchlorate; TDC: Differentiated thyroid cancer; NHANES: National Health and Nutrition Examination Survey; KNHANES: Korean National Health and Nutrition Examination Survey.

\section{Supplementary Information}

The online version contains supplementary material available at https://doi. org/10.1186/s12940-021-00818-8.

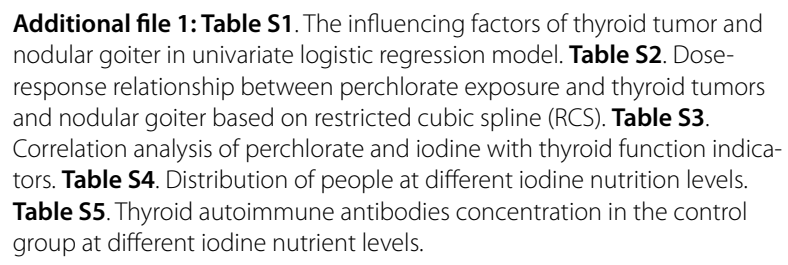
nodular goiter in univariate logistic regression model. Table S2. Doseresponse relationship between perchlorate exposure and thyroid tumors and nodular goiter based on restricted cubic spline (RCS). Table S3. Correlation analysis of perchlorate and iodine with thyroid function indicators. Table S4. Distribution of people at different iodine nutrition levels. Table S5. Thyroid autoimmune antibodies concentration in the control group at different iodine nutrient levels.

\section{Acknowledgements}

We are sincerely grateful to all participants for their support of this study. We also appreciate the help of physicians and technicians from two comprehensive hospitals in Shenzhen.

\section{Authors' contributions}

Wang Huirong: Sample splitting, Urinary iodine test, Writing- Original draft preparation, Conceptualization, Methodology, Software, Formal analysis. Jiang Yousheng: Urinary perchlorate test, Data curation, Resources, Investigation. Song Jiayi: Sample collection, Serum separation, Data Curation, Methodology, Investigation. Liang Huiwen: Sample collection, Sample splitting, Thyroid function indicators tests, Data curation, Investigation. Liu Yuan: Sample collection, Sample splitting, Investigation. Huang Jiewu: Sample splitting, Urinary iodine test. Yin Pengliang: Software, Visualization. Wu Dongting: Investigation. Zhang Hang: Investigation. Liu Xinjie: Resources. Zhou Dongxian: Resources. Wei Wei: Resources. Lei Lin: Resources. Peng Ji: Resources. Zhang Jianqing: Funding acquisition, Supervision, Conceptualization, Writing- Reviewing and Editing. All authors read and approved the final manuscript.

\section{Funding}

This study was supported by the National Key Research and Development Program of China [No. 2017YFC1600500], National Natural Science Foundation of China [No. 22176132], Sanming Project of Medicine in Shenzhen [No. SZSM201811070], and Shenzhen Key Medical Discipline Construction Fund [No. SZXK067].

\section{Availability of data and materials}

The dataset generated during and/or analyzed during the current study are not publicly available due to ethics reason.

\section{Declarations}

\section{Ethics approval and consent to participate}

All participants had signed the Informed Consent Form. The present study has been approved by the Ethics Committee of the Shenzhen Center for Disease Control and Prevention and the two recruiting hospitals ([2019]012A).

\section{Consent for publication}

Not applicable.

\section{Competing interests}

The authors declare that they have no competing interests.

\section{Author details}

${ }^{1}$ Shenzhen Center for Disease Control and Prevention, No.8 Longyuan Road, Nanshan District, Shenzhen 518055, China. ${ }^{2}$ School of Public Health, Southern Medical University, No.1023 Shatai Road, Baiyun District, Guangzhou 510515, China. ${ }^{3}$ Shenzhen Eye Hospital, Shenzhen Key Ophthalmic Laboratory, the Second Affiliated Hospital of Jinan University, No.18 Zetian Road, Futian District, Shenzhen 518040, China. ${ }^{4}$ Shenzhen People's Hospital, No.1017 Dongmen North Road, Luohu District, Shenzhen 518020, China. ${ }^{5}$ Peking University Shenzhen Hospital, No.1120 Lianhua Road, Futian District, Shenzhen 518036, China. ${ }^{6}$ Shenzhen Center for Chronic Disease Control, No.2021 Buxin Road, Luohu District, Shenzhen 518020, China.

Received: 30 September 2021 Accepted: 14 December 2021 Published online: 04 January 2022

\section{References}

1. Durante C, Grani G, Lamartina L, Filetti S, Mandel SJ, Cooper DS. The diagnosis and Management of Thyroid Nodules: a review. JAMA. 2018;319(9):914-24.

2. Ferlay J, Ervik M, Lam F, Colombet M, Mery L, Piñeros M, Znaor A, Soerjomataram I, Bray F: Global Cancer Observatory: Cancer Today. Lyon, France: International Agency for Research on Cancer. In.; 2020. https:// gco.iarc.fr/today (2020). Accessed 10 May 2021.

3. Qian ZJ, Jin MC, Meister KD, Megwalu UC. Pediatric thyroid Cancer incidence and mortality trends in the United States, 1973-2013. JAMA Otolaryngol Head Neck Surg. 2019;145(7):617-23.

4. Mirian C, Grønhøj C, Jensen DH, Jakobsen KK, Karnov K, Jensen JS, et al. Trends in thyroid cancer: retrospective analysis of incidence and survival in Denmark 1980-2014. Cancer Epidemiol. 2018;55:81-7.

5. Wang J, Yu F, Shang Y, Ping Z, Liu L. Thyroid cancer: incidence and mortality trends in China, 2005-2015. Endocrine. 2020;68(1):163-73.

6. Lei L, Shang Q, Liu W, Wang Y, Wang Y, Peng J. Incidences and epidemiologic trends of thyroid Cancer in Shenzhen, 2001 2015. China Cancer. 2019;28(07):504-8 In China.

7. Maniakas A, Davies L, Zafereo ME. Thyroid disease around the world. Otolaryngol Clin N Am. 2018;51(3):631-42.

8. Tang Z, Zhang J, Zhou Q, Xu S, Cai Z, Jiang G. Thyroid Cancer "epidemic": a socio-environmental health problem needs collaborative efforts. Environ Sci Technol. 2020;54(7):3725-7. 
9. Li Q, Yu Y, Wang F, Chen S, Yin Y, Lin H, et al. Urinary perchlorate exposure and risk in women of reproductive age in a fireworks production area of China. Arch Environ Contam Toxicol. 2014;67(1):42-9.

10. Calderón R, Godoy F, Escudey M, Palma P. A review of perchlorate $(\mathrm{ClO}(4)(-))$ occurrence in fruits and vegetables. Environ Monit Assess. 2017;189(2):82

11. Steinmaus CM. Perchlorate in water supplies: sources, exposures, and health effects. Curr Environ Health Rep. 2016;3(2):136-43.

12. Gan Z, Pi L, Li Y, Hu W, Su S, Qin X, et al. Occurrence and exposure evaluation of perchlorate in indoor dust and diverse food from Chengdu, China. Sci Total Environ. 2015;536:288-94.

13. Wu Q, Zhang T, Sun H, Kannan K. Perchlorate in tap water, groundwater, surface waters, and bottled water from China and its association with other inorganic anions and with disinfection byproducts. Arch Environ Contam Toxicol. 2010;58(3):543-50

14. Shi Y, Zhang P, Wang Y, Shi J, Cai Y, Mou S, et al. Perchlorate in sewage sludge, rice, bottled water and milk collected from different areas in China. Environ Int. 2007;33(7):955-62.

15. Eguchi A, Kunisue T, Wu Q, Trang PT, Viet PH, Kannan K, et al. Occurrence of perchlorate and thiocyanate in human serum from e-waste recycling and reference sites in Vietnam: association with thyroid hormone and iodide levels. Arch Environ Contam Toxicol. 2014;67(1):29-41.

16. Leung AM, Pearce EN, Braverman LE. Environmental perchlorate exposure: potential adverse thyroid effects. Curr Opin Endocrinol Diabetes Obes. 2014;21(5):372-6.

17. Dohán O, De la Vieja A, Paroder V, Riedel C, Artani M, Reed M, et al. The sodium/iodide symporter (NIS): characterization, regulation, and medical significance. Endocr Rev. 2003;24(1):48-77.

18. Blount BC, Pirkle JL, Osterloh JD, Valentin-Blasini L, Caldwell KL. Urinary perchlorate and thyroid hormone levels in adolescent and adult men and women living in the United States. Environ Health Perspect. 2006;114(12):1865-71.

19. Steinmaus C, Miller MD, Cushing L, Blount BC, Smith AH. Combined effects of perchlorate, thiocyanate, and iodine on thyroid function in the National Health and nutrition examination survey 2007-08. Environ Res. 2013;123:17-24.

20. Pleus RC, Corey LM. Environmental exposure to perchlorate: a review of toxicology and human health. Toxicol Appl Pharmacol. 2018;358:102-9.

21. Zhang L, Fang C, Liu L, Liu X, Fan S, Li J, et al. A case-control study of urinary levels of iodine, perchlorate and thiocyanate and risk of papillary thyroid cancer. Environ Int. 2018;120:388-93.

22. NCNN: NCCN Clinical Practice Guidelines in Oncology: Thyroid Carcinoma. In.: National Comprehensive Cancer Network; 2018. https:// www.nccn.org/professionals/physician_gls/default.aspx\#thyroid (2018). Accessed 20 May 2021.

23. Hu J, Peng Y, Zheng T, Zhang B, Liu W, Wu C, et al. Effects of trimesterspecific exposure to vanadium on ultrasound measures of fetal growth and birth size: a longitudinal prospective prenatal cohort study. Lancet Planet Health. 2018;2(10):e427-37.

24. Gardell AM, Dillon DM, Smayda LC, von Hippel FA, Cresko WA, Postlethwait $\mathrm{JH}$, et al. Perchlorate exposure does not modulate temporal variation of whole-body thyroid and androgen hormone content in threespine stickleback. Gen Comp Endocrinol. 2015;219:45-52.

25. York RG, Lewis E, Brown WR, Girard MF, Mattie DR, Funk KA, et al. Refining the effects observed in a developmental neurobehavioral study of ammonium perchlorate administered orally in drinking water to rats. I. Thyroid and reproductive effects. Int J Toxicol. 2005;24(6):403-18.

26. Wang K, Xu J, Li S, Liu S, Zhang L. Population-based study evaluating and predicting the probability of death resulting from thyroid cancer among patients with papillary thyroid microcarcinoma. Cancer Med. 2019;8(16):6977-85.

27. Mijović T, How J, Pakdaman M, Rochon L, Gologan O, Hier MP, et al. Body mass index in the evaluation of thyroid cancer risk. Thyroid. 2009;19(5):467-72.

28. Choi SW, Ryu SY, Han MA, Park J. The association between the socioeconomic status and thyroid cancer prevalence; based on the Korean National Health and nutrition examination survey 2010-2011. J Korean Med Sci. 2013;28(12):1734-40.
29. Schmid D, Ricci C, Behrens G, Leitzmann MF. Adiposity and risk of thyroid cancer: a systematic review and meta-analysis. Obes Rev. 2015;16(12):1042-54.

30. Yeo Y, Shin DW, Han KD, Kim D, Kim TH, Chun S, et al. Smoking, alcohol consumption, and the risk of thyroid cancer: a population-based Korean cohort study of 10 million people. Thyroid. 2020. https://doi. org/10.1089/thy.2019.0508.

31. Horton MK, Blount BC, Valentin-Blasini L, Wapner R, Whyatt R, Gennings $C$, et al. CO-occurring exposure to perchlorate, nitrate and thiocyanate alters thyroid function in healthy pregnant women. Environ Res. 2015;143(Pt A):1-9.

32. Siglin JC, Mattie DR, Dodd DE, Hildebrandt PK, Baker WH. A 90-day drinking water toxicity study in rats of the environmental contaminant ammonium perchlorate. Toxicol Sci. 2000;57(1):61-74.

33. Lawrence JE, Lamm SH, Pino S, Richman K, Braverman LE. The effect of short-term low-dose perchlorate on various aspects of thyroid function. Thyroid. 2000;10(8):659-63.

34. Braverman LE, Pearce EN, He X, Pino S, Seeley M, Beck B, et al. Effects of six months of daily low-dose perchlorate exposure on thyroid function in healthy volunteers. J Clin Endocrinol Metab. 2006;91(7):2721-4.

35. Haymart MR, Repplinger DJ, Leverson GE, Elson DF, Sippel RS, Jaume JC, et al. Higher serum thyroid stimulating hormone level in thyroid nodule patients is associated with greater risks of differentiated thyroid cancer and advanced tumor stage. J Clin Endocrinol Metab. 2008;93(3):809-14

36. Wu X, Lun Y, Jiang H, Gang Q, Xin S, Duan Z, et al. Coexistence of thyroglobulin antibodies and thyroid peroxidase antibodies correlates with elevated thyroid-stimulating hormone level and advanced tumor stage of papillary thyroid cancer. Endocrine. 2014;46(3):554-60.

37. Hoffmann S, Hofbauer LC, Scharrenbach V, Wunderlich A, Hassan I, Lingelbach S, et al. Thyrotropin (TSH)-induced production of vascular endothelial growth factor in thyroid cancer cells in vitro: evaluation of TSH signal transduction and of angiogenesis-stimulating growth factors. J Clin Endocrinol Metab. 2004;89(12):6139-45.

38. McLachlan SM, Rapoport B. Why measure thyroglobulin autoantibodies rather than thyroid peroxidase autoantibodies? Thyroid. 2004; 14(7):510-20.

39. Wang F, Wang Y, Wang L, Wang $X$, Sun C, Xing M, et al. Strong association of high urinary iodine with thyroid nodule and papillary thyroid cancer. Tumour Biol. 2014;35(11):11375-9.

40. Yang X, Sun J, Han J, Sun L, Wang H, Zhang D, et al. lodine promotes thyroid cancer development via SPANXA1 through the PI3K/AKT signalling pathway. Oncol Lett. 2019;18(1):637-44.

41. Zimmermann MB, Galetti V. lodine intake as a risk factor for thyroid cancer: a comprehensive review of animal and human studies. Thyroid Res. 2015:8(1):8.

42. Xiu C, He Q, Zhao HJ, Yuan ZN, Guo LH, Wang FQ, et al. Strong correlation of abnormal serum and urinary iodine levels with papillary thyroid Cancer: a case-control study. Biomed Environ Sci. 2020;33(1):62-7.

43. Zhao H, Li H, Huang T. High iodine intake and central lymph node metastasis risk of papillary thyroid cancer. J Trace Elem Med Biol. 2019;53:16-21.

44. Wang D, Yu S, Yin Y, Xie S, Cheng Q, Li H, et al. lodine status of euthyroid adults: a cross-sectional, multicenter study. J Clin Lab Anal. 2019;33(4):e22837.

45. Soldin OP, Tractenberg RE, Pezzullo JC. Do thyroxine and thyroid-stimulating hormone levels reflect urinary iodine concentrations? Ther Drug Monit. 2005;27(2):178-85.

46. Gostas DE, Larson-Meyer DE, Yoder HA, Huffman AE, Johnson EC. Dietary relationship with $24 \mathrm{~h}$ urinary iodine concentrations of Young adults in the mountain west region of the United States. Nutrients. 2020;12(1):121.

47. O'Kane SM, Mulhern MS, Pourshahidi LK, Strain JJ, Yeates AJ. Micronutrients, iodine status and concentrations of thyroid hormones: a systematic review. Nutr Rev. 2018;76(6):418-31.

48. Wang B, He W, Li Q, Jia X, Yao Q, Song R, et al. U-shaped relationship between iodine status and thyroid autoimmunity risk in adults. Eur J Endocrinol. 2019;181(3):255-66.

49. Latrofa F, Fiore E, Rago T, Antonangeli L, Montanelli L, Ricci D, et al. lodine contributes to thyroid autoimmunity in humans by 
unmasking a cryptic epitope on thyroglobulin. J Clin Endocrinol Metab. 2013;98(11):E1768-74.

50. Pearce EN, Caldwell KL. Urinary iodine, thyroid function, and thyroglobulin as biomarkers of iodine status. Am J Clin Nutr. 2016;104 Suppl 3(Suppl 3):898S-901S.

51. Huber DR, Blount BC, Mage DT, Letkiewicz FJ, Kumar A, Allen RH. Estimating perchlorate exposure from food and tap water based on US biomonitoring and occurrence data. J Expo Sci Environ Epidemiol. 2011;21(4):395-407

\section{Publisher's Note}

Springer Nature remains neutral with regard to jurisdictional claims in published maps and institutional affiliations.

- fast, convenient online submission

- thorough peer review by experienced researchers in your field

- rapid publication on acceptance

- support for research data, including large and complex data types

- gold Open Access which fosters wider collaboration and increased citations

- maximum visibility for your research: over $100 \mathrm{M}$ website views per year

At BMC, research is always in progress.

Learn more biomedcentral.com/submissions 